\section{Differential expression of the leptin receptor in cancers of the breast.}
Shahan Mamoor, MS ${ }^{1}$
$3 \quad$ shahanmamoor@gmail.com
East Islip, NY 11730
Breast cancer affects women at relatively high frequency ${ }^{1}$. We mined published microarray datasets ${ }^{2,3}$ to determine in an unbiased fashion and at the systems level genes most differentially expressed in the primary tumors of patients with breast cancer. We report here significant differential expression of the gene encoding the leptin receptor, LEPR, when comparing primary tumors of the breast to the tissue of origin, the normal breast. LEPR was also differentially expressed in the tumor cells of patients with triple negative breast cancer. LEPR mRNA was present at significantly lower quantities in tumors of the breast as compared to normal breast tissue. Analysis of human survival data revealed that expression of LEPR in primary tumors of the breast was correlated with overall survival in patients with luminal A subtype cancers, demonstrating a relationship between primary tumor expression of a differentially expressed gene and patient survival outcomes influenced by molecular subtype. LEPR may be of relevance to initiation, maintenance or progression of cancers of the female breast.

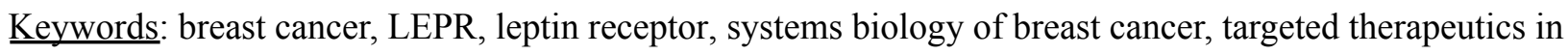
breast cancer. 
Invasive breast cancer is diagnosed in over a quarter of a million women in the United States each year $^{1}$ and in 2018 , breast cancer was the leading cause of cancer death in women worldwide ${ }^{4}$. While patients with localized breast cancer are provided a 99\% 5-year survival rate, patients with regional breast cancer, cancer that has spread to lymph nodes or nearby structures, are provided an $86 \% 5$-year survival rate $^{5,6}$. Patients with metastasis to distant sites, like the brain, are provided a $27 \% 5$-year survival rate ${ }^{5,6}$. Understanding how primary tumors are most transcriptionally different from the tissue from which they originate, the breast, can facilitate development of novel diagnostic and therapeutics to promote early detection and enhanced treatment, and contribute to efforts to prevent progression to metastatic stages. We mined published microarray data ${ }^{2,3}$ to understand at the transcriptome level and in an unbiased fashion genes most differentially expressed in primary tumors of the breast as compared to normal breast tissue. leptin receptor emerged as among the most differentially expressed genes in cancer of the female breast.

\section{Methods}

We utilized datasets GSE109169 and GSE38959 $9^{3}$ for this differential gene expression analysis of female breast cancer. GSE109169 was generated using Affymetrix Human Exon 1.0 ST Array technology with $n=25$ normal breast tissue and $n=25$ tumors of the breast; analysis was performed using platform GPL5175. The tissues from this first dataset are paired tissues (25 tumors matching 25 breast tissues from 25 patients). GSE38959 was generated using Agilent-014850 Whole Human Genome Microarray 4x44K G4112F technology with $n=13$ samples of normal mammary gland ductal cells and $n=30$ samples of tumor cells from patients with triple negative breast cancer; analysis was performed using platform GPL4133. The Benjamini and Hochberg method of p-value adjustment was used for ranking of differential expression but raw $p$-values were used to assess statistical significance of global differential expression. Log-transformation of data was auto-detected, and the NCBI generated category of platform annotation was used. A statistical test was performed to evaluate whether LEPR expression was significantly different between primary breast tumors and breast tissue using a two-tailed t-test. For Kaplan-Meier survival analysis, we used the Kaplan-Meier plotter online tool ${ }^{7}$ for correlation of LEPR mRNA expression levels with overall survival (OS) in $n=404$ patients with basal subtype cancer, $n=794$ patients with luminal A subtype cancer, $n=515$ patients with luminal B subtype cancer, and $n=166$ patients with HER2+ cancer.

\section{Results}

We performed discovery of genes associated with breast cancer in females by mining two independently published microarray datasets ${ }^{2,3}$.

\section{LEPR is differentially expressed in primary tumors of the breast.}

Comparison of 25 normal breast tissues to 25 tumors of the breast ${ }^{2}$ from patients with early-onset breast cancer revealed that the gene encoding the leptin receptor, LEPR, was among the genes most differentially expressed in tumors of the breast in human breast cancer (Chart 1). When sorting each of the genes expressed in tumors of the breast based on significance of difference as compared to normal breast tissue, LEPR ranked 28 out of 19076 total transcripts, equating to $99.9 \%$ differential expression (Chart 1). Differential expression of LEPR in female breast cancer was statistically significant (Chart 1; $p=3.97 \mathrm{E}-18$ ).

Analysis of a second microarray datase ${ }^{3}$, here in tumor cells of patients with triple negative breast cancer as compared to normal mammary gland ductal cells, again revealed significant differential expression of LEPR in human breast cancer (Chart 2). When sorting each of the genes expressed in the tumor cells of patients with triple negative breast cancer based on significance of difference as compared 
to normal mammary gland ductal cells, LEPR ranked 7959 out of 45015 total transcripts, equating to 82.3\% differential expression (Chart 2). Differential expression of LEPR in the tumor cells of patients with triple negative breast cancer was statistically significant (Chart $2 ; p=3.47 \mathrm{E}-03$ ). These data suggested that differential expression of LEPR was not an artifact of a single microarray dataset, nor was it associated with one type of breast cancer, rather a general feature of cancers of the breast.

\section{LEPR is expressed at significantly lower levels in breast tumors as compared to the breast.}

We obtained exact mRNA expression levels for LEPR from the breast and from breast tumors to understand the magnitude and direction of LEPR expression change. LEPR was expressed at lower levels in tumors of the breast as compared to normal breast tissue (Figure 1). Decreased expression of LEPR in primary breast tumors was statistically significant (Figure 1: $p<0.0001$ ). LEPR was expressed at $7.96 \pm$ 0.41 arbitrary units (AU) in normal breast tissue, while it was expressed at $6.40 \pm 0.43 \mathrm{AU}$ in tumors of the breast. We calculated a mean fold change of 0.80 in LEPR mRNA levels when comparing primary tumors of the breast to normal breast tissues.

\section{LEPR expression correlates with survival outcomes in luminal A subtype human breast cancer.}

We performed Kaplan-Meier survival analysis in an attempt to correlate LEPR mRNA expression levels with survival outcomes in patients with breast cancer, evaluating correlations between molecular subtype and overall survival. We observed a correlation between LEPR expression and overall survival (OS) in patients with luminal A subtype breast cancer which approached statistical significance (Figure 2; $\log$ rank $p$-value: 0.054 for overall survival, hazard ratio: 0.73 (0.53-1.01) (Fig. 2)). LEPR mRNA levels were a positive prognostic indicator in luminal A subtype breast cancer patients. Median OS was 125.92 months for luminal A patients with low tumor expression of LEPR while median OS was 151.04 months for luminal A patients with high tumor expression of LEPR (Chart 3). LEPR primary tumor expression was not correlated with overall survival in basal subtype (Figure 2; log rank $p$-value: 0.2 for OS, hazard ratio: 1.29 (0.88-1.88) (Fig. 2), luminal B subtype (Figure 2; log rank $p$-value: 0.65 for OS, hazard ratio: 0.92 (0.65-1.31) (Fig. 2) or in HER2+ breast cancer (Figure 2; log rank $p$-value: 0.41 for OS, hazard ratio: 0.79 (0.45-1.4) (Fig. 2)).

Thus, through comparative transcriptome analysis of primary tumors of the breast and normal breast tissue, we found that differential expression and down-regulation of LEPR was among the most significant transcriptional features in primary tumors from patients with breast cancer. LEPR expression in primary tumors of the breast was correlated with overall survival in patients with luminal A disease, with mRNA levels of LEPR a positive prognostic indicator for luminal A subtype breast cancer patients.

\section{Discussion}

Invasive breast cancer is a medical problem with a $27 \% 5$-year survival rate for women whose disease has spread to distant sites ${ }^{5,6}$. To facilitate understanding of the basic transcriptional differences between primary tumors of the breast and the tissues from which these tumors originate, normal breast tissues, we performed comparative transcriptome analysis using two independently published microarray datasets $^{2,3}$, providing evidence here that differential expression of the leptin receptor, encoded by LEPR, is a defining transcriptional feature of human breast cancer: in early onset-breast cancer, and in patients diagnosed after age 50. LEPR was expressed at significantly lower levels in primary tumors from patients with breast cancer as compared to normal breast tissue. Importantly, in patients with luminal A breast cancers, expression of LEPR was correlated with overall survival. Leptin receptor, and the molecular processes to which it pertains to, may be relevant to the initiation or progression of human breast cancer. 


\section{References}

1. DeSantis, C.E., Ma, J., Goding Sauer, A., Newman, L.A. and Jemal, A., 2017. Breast cancer statistics, 2017, racial disparity in mortality by state. CA: a cancer journal for clinicians, 67(6), pp.439-448.

2. Chang, J.W., Kuo, W.H., Lin, C.M., Chen, W.L., Chan, S.H., Chiu, M.F., Chang, I.S., Jiang, S.S., Tsai, F.Y., Chen, C.H. and Huang, P.H., 2018. Wild-type p53 upregulates an early onset breast cancer-associated gene GAS7 to suppress metastasis via GAS7-CYFIP1- mediated signaling pathway. Oncogene, 37(30), pp.4137-4150.

3. Komatsu, M., Yoshimaru, T., Matsuo, T., Kiyotani, K., Miyoshi, Y., Tanahashi, T., Rokutan, K., Yamaguchi, R., Saito, A., Imoto, S. and Miyano, S., 2013. Molecular features of triple negative breast cancer cells by genome-wide gene expression profiling analysis. International journal of oncology, 42(2), pp.478-506.

4. Bray, F., Ferlay, J., Soerjomataram, I., Siegel, R.L., Torre, L.A. and Jemal, A., 2018. Global cancer statistics 2018: GLOBOCAN estimates of incidence and mortality worldwide for 36 cancers in 185 countries. CA: a cancer journal for clinicians, 68(6), pp.394-424.

5. ACS Cancer Facts \& Figures 2019.

https://www.cancer.net/cancer-types/breast-cancermetastatic/statistics.

6. Survival Rates for Breast Cancer. https://www.cancer.org/cancer/breast-cancer/ understanding-a-breast-cancer-diagnosis/breast-cancer-survival-rates.html

7. Györffy, B., Lanczky, A., Eklund, A.C., Denkert, C., Budczies, J., Li, Q. and Szallasi, Z., 2010. An online survival analysis tool to rapidly assess the effect of 22,277 genes on breast cancer prognosis using microarray data of 1,809 patients. Breast cancer research and treatment, 123(3), pp.725-731. 


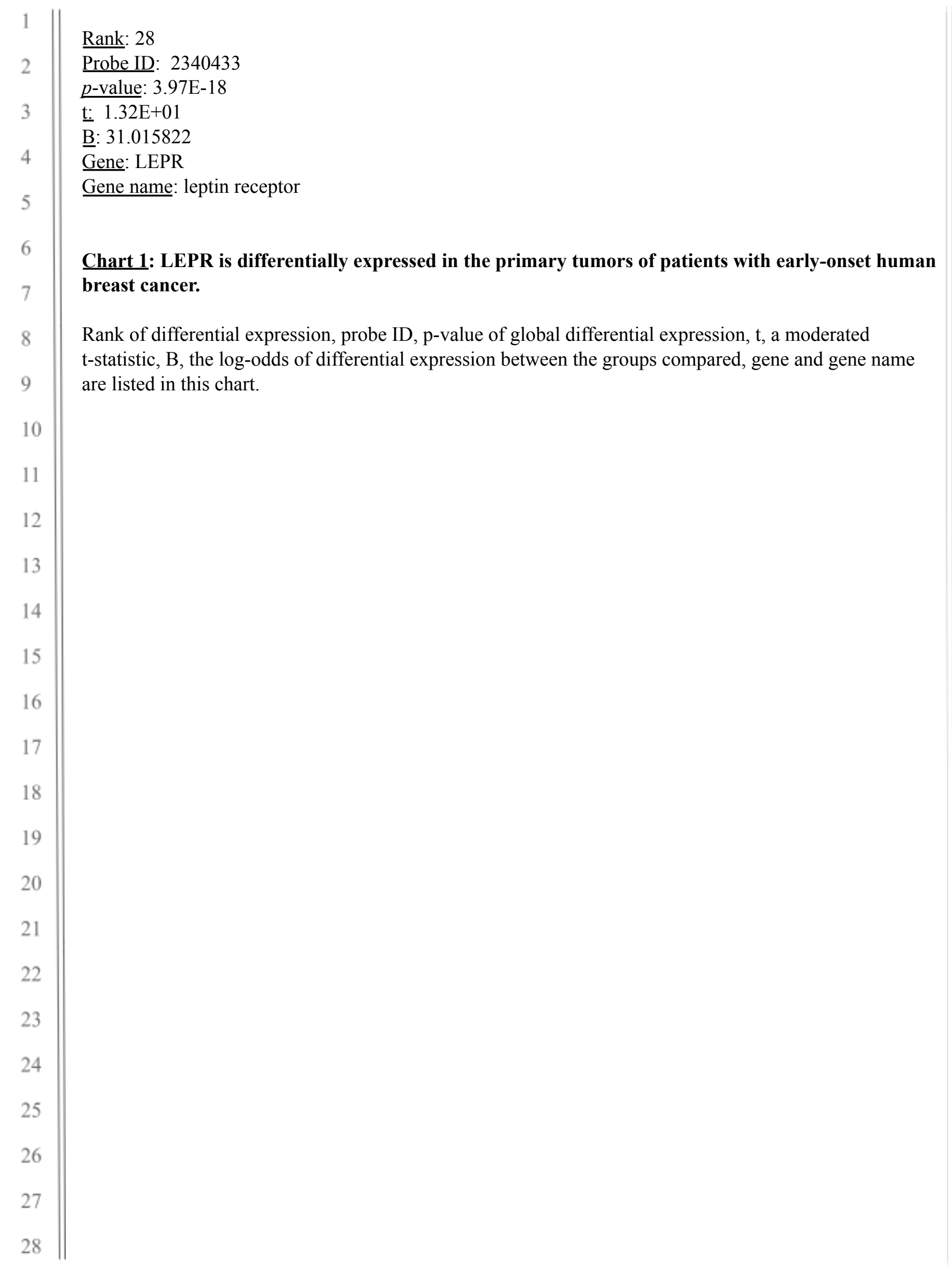




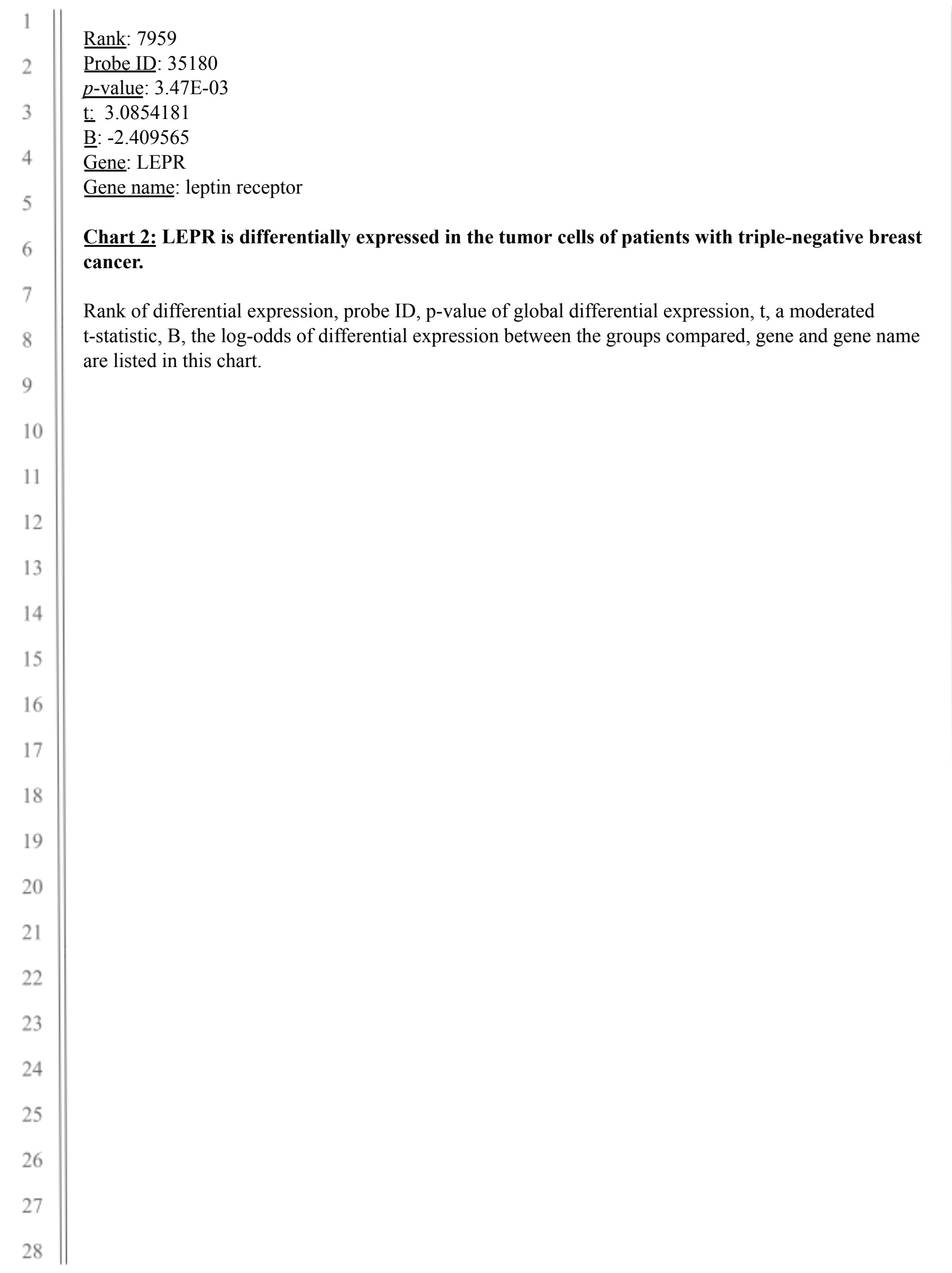




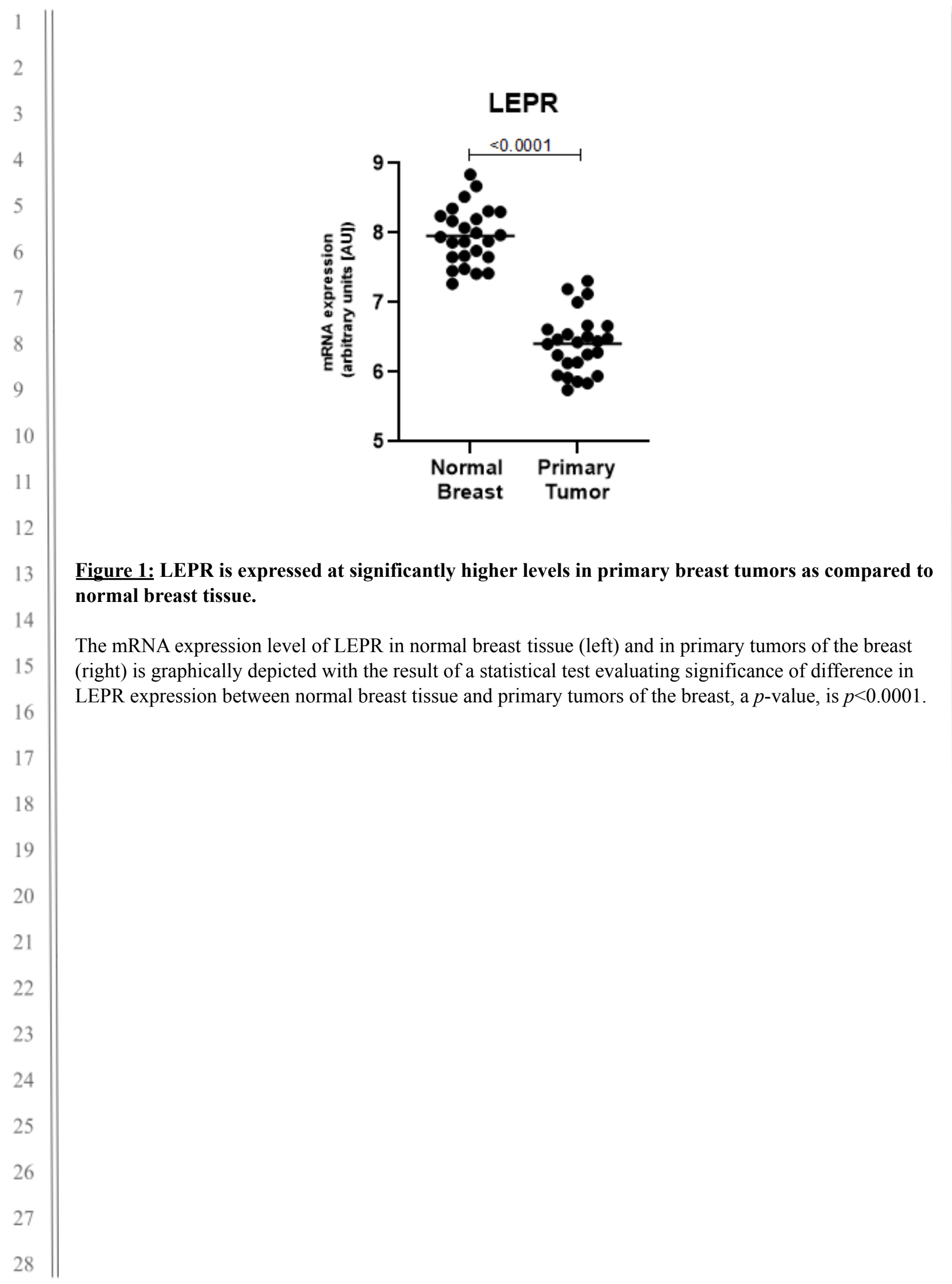



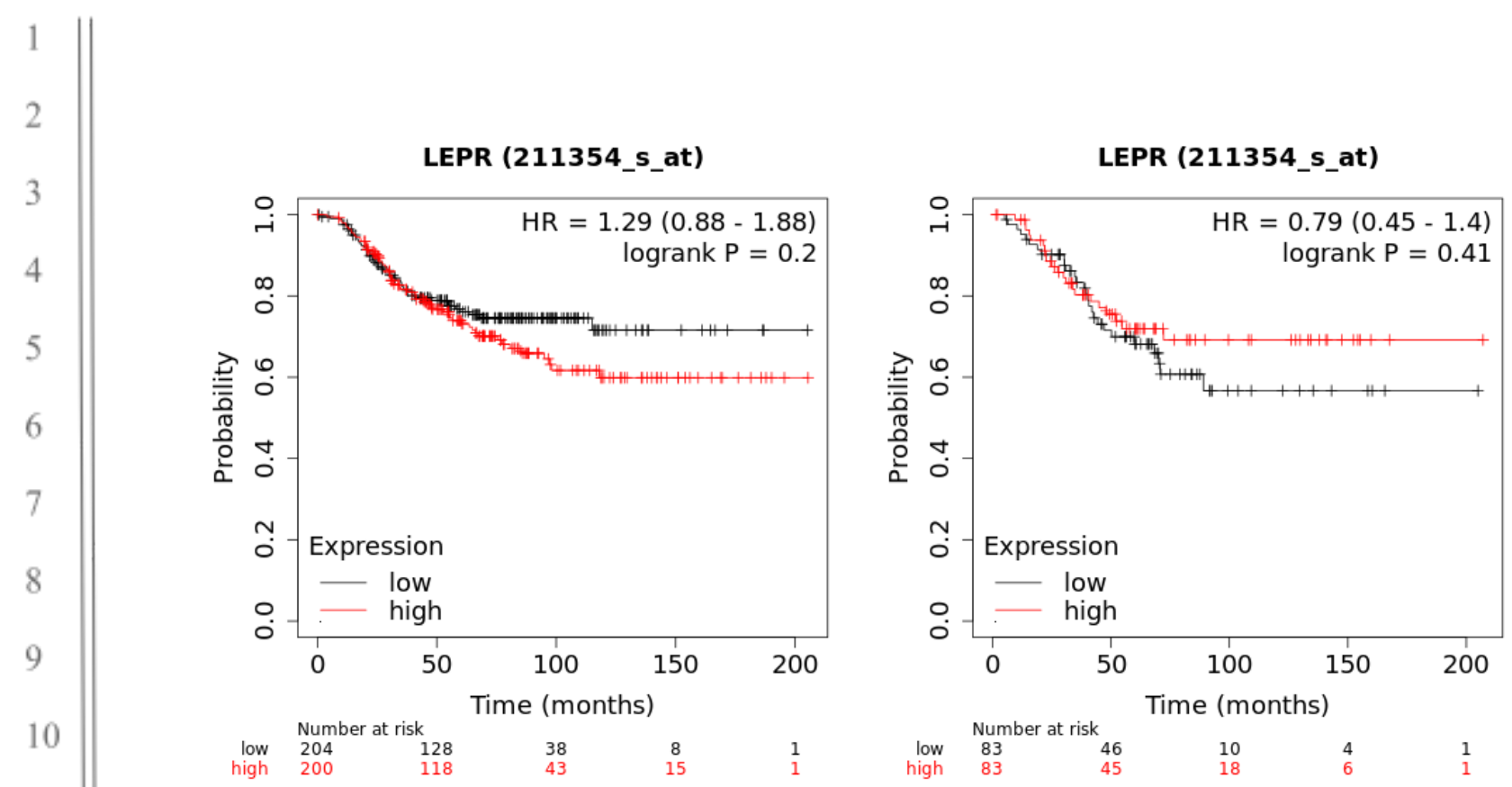

LEPR (211354_s_at)

Figure 2: LEPR expression correlates with overall survival in patients with luminal A subtype breast cancer, but not in patients with basal, luminal B or HER2+ cancer.

Depicted in this Kaplan-Meier plot is the probability of overall survival (OS) for $n=404$ patients with basal subtype breast cancer (upper left), $n=166$ patients with HER2 + breast cancer (upper right), $n=794$ patients with luminal A breast cancer (lower left), and $n=515$ patients with luminal B breast cancer (lower right), stratified into two groups, based on low or high expression of LEPR in patient primary tumors. The log rank p-value denoting statistical significance of difference in overall survival when comparing the two groups, as well as hazard ratio for this comparison is listed above. Listed below is the number of patients at risk (number of patients alive) per interval, after stratification based on LEPR expression; in the first interval, number at risk is number of patients alive; in each subsequent interval, number at risk is the number at risk less those who have expired or are censored. 


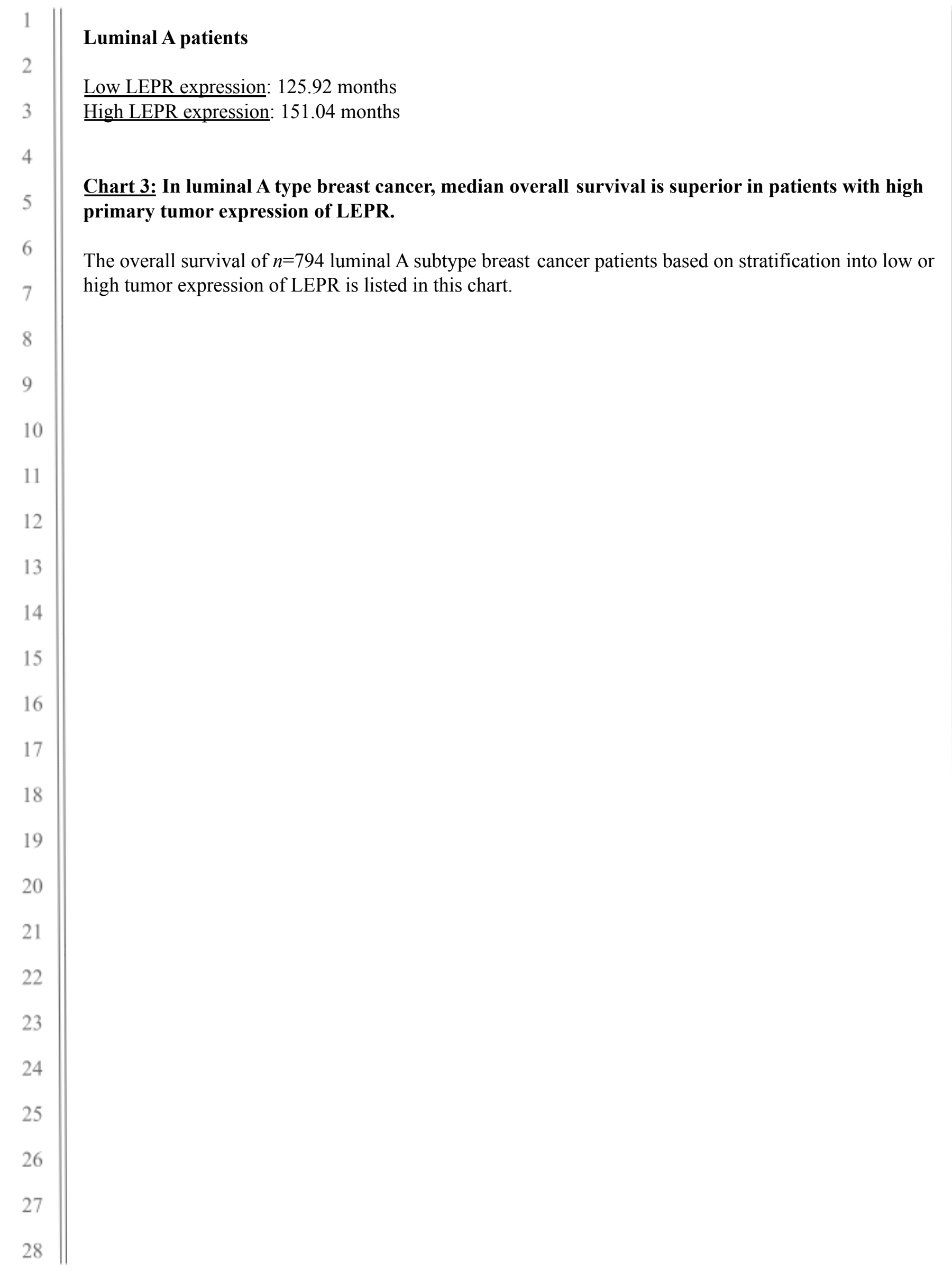

